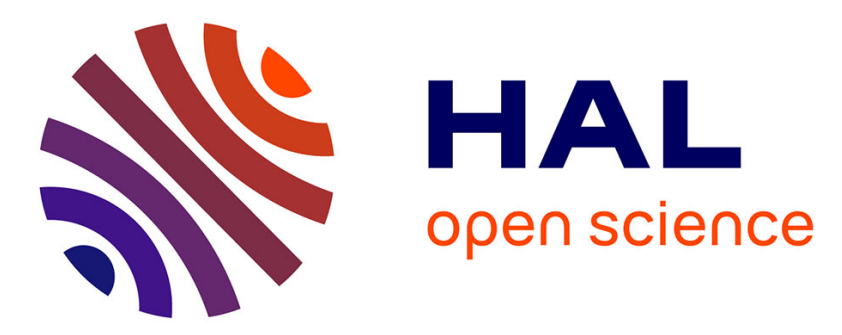

\title{
A dynamical treatment of isobaric widths in fission: An example of frozen quantal fluctuations
}

\author{
H. Nifenecker
}

\section{To cite this version:}

H. Nifenecker. A dynamical treatment of isobaric widths in fission: An example of frozen quantal fluctuations. Journal de Physique Lettres, 1980, 41 (3), pp.47-51. 10.1051/jphyslet:0198000410304700 . jpa-00231719

\section{HAL Id: jpa-00231719 https://hal.science/jpa-00231719}

Submitted on 1 Jan 1980

HAL is a multi-disciplinary open access archive for the deposit and dissemination of scientific research documents, whether they are published or not. The documents may come from teaching and research institutions in France or abroad, or from public or private research centers.
L'archive ouverte pluridisciplinaire HAL, est destinée au dépôt et à la diffusion de documents scientifiques de niveau recherche, publiés ou non, émanant des établissements d'enseignement et de recherche français ou étrangers, des laboratoires publics ou privés. 


\title{
LE JOURNAL DE PHYSIQUE-LETTRES
}

Classification

Physics Abstracts

$25.85-25.80$

\section{A dynamical treatment of isobaric widths in fission : An example of frozen quantal fluctuations}

\author{
H. Nifenecker \\ Centre d'Etudes Nucléaires de Grenoble, Département de Recherche Fondamentale, \\ Laboratoire de Chimie Physique Nucléaire, 85X, 38041 Grenoble Cedex, France
}

(Resule 19 novembre 1979, acceptéle ll décembre 1979)

\begin{abstract}
Résumé. - On explique les largeurs des distributions isobariques des fragments de fission comme résultant des fluctuations dans le fondamental d'un oscillateur harmonique dépendant du temps dans le mode d'équilibration de charge. On trouve que les valeurs expérimentales sont reproduites pour des durées de la phase de striction du col reliant les fragments naissants d'environ $2 \times 10^{-21} \mathrm{~s}$. L'extension du modèle aux réactions induites par ions est discutée.
\end{abstract}

\begin{abstract}
We treat the isobaric distributions observed in the fission process as the result of zero-point oscillations of a time dependent harmonic oscillator in the charge equilibration mode. It is found that the experimental values of these widths can be reproduced assuming a necking time around $2 \times 10^{-21} \mathrm{~s}$. Possible extension of the model to heavy ion reactions is discussed.
\end{abstract}

1. Introduction. - The interest of the charge equilibration mode has been recently underlined in heavy ion reactions [1], [2], [3], [4].

Experimentally it appears [1] that the proton to neutron degree of freedom is probably the first to reach equilibrium in deep inelastic reactions. This is true, at least, for the average value of the proton to neutron ratio. The short equilibration time was related [2] to the high frequency of the giant dipole resonance mode. Extrapolating the observed energies of the giant dipole resonance to the very elongated dinuclear shapes involved in heavy ion collisions one gets typical equilibration time (a quarter of a period) of around $10^{-22} \mathrm{~s}$. This approach underlines the collective character of the charge equilibration mode. This collectivity has been recently questioned by L. Moretto [5] on the grounds that the high excitation energies involved in heavy ion reactions should dissolve all collective states in a continuum. This argument should not hold in the case of low energy nuclear fission since the excitation energies involved at scission are only of the order of $10 \mathrm{MeV}$ [6] ; moreover one knows that the number of pairs broken during the fission process is small [7] which implies a high degree of collectivity of the nucleonic motion. Therefore in the following, we try to estimate the extent to which fission fragments isobaric distributions reflect quantal fluctuations.
2. The model. - We describe very schematically the fissionning nucleus by two overlapping spheres, with radii $R_{1}$ and $R_{2}$ varying in time in order to obey volume conservation. The radius of the aperture through with the two spheres may exchange matter is equal to $c$. The masses and charges of the two nascent fragments are $A_{1}, A_{2}=A_{T}-A_{1}, Z$ and $Z_{T}-Z$ respectively assuming the fission of a nucleus with mass $A_{T}$, charge $Z_{T}$. Such a parametrization is, for example, used by Brosa and Krappe [8]. As usual it is assumed that the proton to neutron degree of freedom is much faster than the mass one. Therefore it is possible to let the masses fixed and study the variations of the $Z$ and $c$ parameters alone.

We suppose, as customarily done, that the motion along the $Z$ coordinate is harmonic with potential

$$
V(Z)=\frac{1}{2} K\left(Z-Z_{\mathrm{p}}\right)^{2}
$$

where $K$ is the stiffness constant of the harmonic oscillator and $Z_{\mathrm{p}}$ the most probable charge. The kinetic energy reads

$$
\frac{1}{2} m\left(\frac{\mathrm{d} Z}{\mathrm{~d} t}\right)^{2}
$$

At variance with preceding treatments we want to take explicitly into account the variations of the 
oscillator parameters with time. It is clear that, near scission, the relevant quantity most affected by the shrinking of the neck radius $c$ is the inertia parameter $m$ since it diverges when $c$ approaches zero. In the following we may therefore, in first approximation, neglect the variations of both $K$ and $Z_{\mathrm{p}}$ with time.

For the stiffness constant we use, as in ref. [7], the value given by Berlanger et al. [9] namely

$K \mathrm{MeV} /(\text { charge unit })^{2}=1.39\left(A_{1}^{-1 / 3}+A_{2}^{-1 / 3}\right)+$

$$
+186.28\left(\frac{1}{A_{1}}+\frac{1}{A_{2}}\right)-\frac{2.88}{1.24\left(A_{1}^{1 / 3}+A_{2}^{1 / 3}\right)} .
$$

Also, as in ref. [7], we use for $m$ the expression derived by Brosa and Krappe [8]

$$
m=\frac{2 \pi}{3} r_{0}^{3} m \frac{A_{T}^{2}}{Z_{T} N_{T}} \frac{1}{c}
$$

where $A_{T}=A_{1}+A_{2}, Z_{T}$ and $N_{T}$ are the charge and neutron number of the fissioning species, $m$ is the average nucleonic mass, $r_{0}=1.16 \mathrm{Fm}$.

The inertia $m$ is time dependent through the neck radius $c$.

The time dependent Hamiltonian is therefore

$$
H(t)=\frac{p^{2}}{2 m(t)}+\frac{K q^{2}}{2}
$$

where we have written

$$
\begin{aligned}
& q=Z-Z_{\mathrm{p}} \\
& p=\frac{(\hbar)}{i} \frac{\mathrm{d}}{\mathrm{d} q} .
\end{aligned}
$$

3. Time-evolution equation for isobaric width. Since we are interested in the width of the isobaric distribution it is not necessary to solve the full time dependent Schrödinger equation. It is enough to consider the time evolution of the first two moments $\langle q\rangle$ and $\left\langle q^{2}\right\rangle$ of the charge distributions. The time-evolution equations may be obtained from the Ehrenfest theorem which expresses the time derivative of the mean value of any observable $A$

$$
i \hbar \frac{\mathrm{d}\langle A\rangle}{\mathrm{d} t}=[A, H]+i \hbar \frac{\partial A}{\partial t} .
$$

We consider the observables $p, p^{2}, q, q^{2}$ which do not explicitly depend on time.

A first set of equations relate $\langle p\rangle$ and $\langle q\rangle$, namely

$$
\begin{aligned}
& \frac{\mathrm{d}}{\mathrm{d} t}\langle q\rangle=\frac{\langle p\rangle}{m(t)} \\
& \frac{\mathrm{d}}{\mathrm{d} t}\langle p\rangle=-K\langle q\rangle .
\end{aligned}
$$

The solution $\langle p\rangle_{t}=\langle q\rangle_{t}=0$ is obtained if at time $t=0$ we choose $\langle p\rangle_{t=0}=\langle q\rangle_{t=0}=0$.

This means that the initial state is assumed to be equilibrated. Furthermore the stability of $\langle p\rangle$ and $\langle q\rangle$ is related to our assumption that $Z_{\mathbf{p}}$ did not vary with time. Under these conditions

$$
\begin{aligned}
& \sigma^{2}(p)=\left\langle p^{2}\right\rangle \\
& \sigma^{2}(q)=\left\langle q^{2}\right\rangle .
\end{aligned}
$$

Application of the Ehrenfest equation then gives

$$
\begin{aligned}
m \frac{\mathrm{d}}{\mathrm{d} t} \sigma^{2}(q) & =\langle p q+q p\rangle \\
\frac{\mathrm{d}}{\mathrm{d} t} \sigma^{2}(p) & =-m K \frac{\mathrm{d}}{\mathrm{d} t} \sigma^{2}(q) \\
\frac{\mathrm{d}}{\mathrm{d} t}\langle p q+q p\rangle & =2\left[\frac{1}{m} \sigma^{2}(p)-K \sigma^{2}(q)\right] .
\end{aligned}
$$

From (3.1) and (3.3) one obtains

$$
\frac{\mathrm{d}}{\mathrm{d} t} m \frac{\mathrm{d}}{\mathrm{d} t} \sigma^{2}(q)=2\left[\frac{1}{m} \sigma^{2}(p)-K \sigma^{2}(q)\right]
$$

and by further differentiation

$$
\begin{aligned}
\frac{\mathrm{d}}{\mathrm{d} t} m \frac{\mathrm{d}}{\mathrm{d} t} m \frac{\mathrm{d}}{\mathrm{d} t} \sigma^{2}(q) & =2 \frac{\mathrm{d}}{\mathrm{d} t} \sigma^{2}(p)-2 K \frac{\mathrm{d}}{\mathrm{d} t} m \sigma^{2}(q) \\
& =-4 m K \frac{\mathrm{d}}{\mathrm{d} t} \sigma^{2}(q)-2 K \frac{\mathrm{d} m}{\mathrm{~d} t} \sigma^{2}(q) .
\end{aligned}
$$

After setting

$$
\begin{aligned}
y & =\sigma^{2}(q) \\
\Omega(t) & =\omega^{2}(t)=\frac{K}{m(t)}
\end{aligned}
$$

and integrating once, one is led to the integro differential equation

$$
\begin{aligned}
& \frac{\mathrm{d}^{2}}{\mathrm{~d} t^{2}} y-\frac{1}{\Omega} \frac{\mathrm{d} \Omega}{\mathrm{d} t} \frac{\mathrm{d}}{\mathrm{d} t} y+4 \Omega y= \\
& =\frac{a \Omega^{2}}{K^{2}}-2 \Omega^{2} \int_{0}^{t} \frac{y}{\Omega^{2}} \frac{\mathrm{d} \Omega}{\mathrm{d} u} \mathrm{~d} u
\end{aligned}
$$

where $a$ is a constant obtained from the initial conditions

$$
\begin{array}{r}
\frac{a}{K^{2}} \Omega_{t=0}^{2}=\left|\frac{\mathrm{d}^{2}}{\mathrm{~d} t^{2}} y\right|_{t=0}-\frac{1}{\Omega_{t=0}}\left|\frac{\mathrm{d} \Omega}{\mathrm{d} t}\right|_{t=0}\left|\frac{\mathrm{d}}{\mathrm{d} t} y\right|_{t=0}+ \\
+4 \Omega_{t=0} y_{t=0} .
\end{array}
$$

In the time independent case $a \frac{\Omega^{2}}{K^{2}}$ has a simple meaning since it is well known [10] that

$$
\frac{\mathrm{d}^{2}}{\mathrm{~d} t^{2}} y+4 \Omega y=\frac{4 \varepsilon}{m}
$$


we therefore write in a similar way

$$
a \frac{\Omega^{2}}{K^{2}}=4 \varepsilon_{0} \frac{\Omega}{K} \times\left(\frac{\Omega}{\Omega_{0}}\right) .
$$

For slow enough initial motion one must have, as in the time independent harmonic oscillator case

$$
\frac{K^{2}}{4 \Omega}\left|\frac{\mathrm{d} y}{\mathrm{~d} t}\right|_{0}^{2}+\frac{K^{2}}{16 \Omega^{2}}\left|\frac{\mathrm{d}^{2} y}{\mathrm{~d} t^{2}}\right|_{0}^{2} \leqslant \varepsilon_{0}^{2}-\frac{\hbar^{2}}{4} \Omega
$$

which means that the initial motion as well as the subsequent oscillatory one must obey the Heisenberg uncertainty relationship.

For a discussion of equation (3.4) it is convenient to set

$$
y=Z \omega(t)
$$

then the differential equation for $Z$ writes with

$$
\begin{gathered}
T=\frac{2 \pi}{\omega} \\
\frac{\mathrm{d}^{2} Z}{\mathrm{~d} t^{2}}+\frac{Z}{T} \frac{\mathrm{d}^{2} T}{\mathrm{~d} t^{2}}+\frac{4 \omega^{2}}{T} \int_{0}^{t} T \frac{\mathrm{d} Z}{\mathrm{~d} u} \mathrm{~d} u=0
\end{gathered}
$$

if $\frac{\mathrm{d}^{2} T}{\mathrm{~d} t^{2}}=0$ then

$$
Z=Z_{0}=0 \text { is a solution }
$$

We refer to it as the " adiabatic solution".

Since

$$
\frac{\mathrm{d}^{2} T}{\mathrm{~d} t^{2}}=\frac{4 \pi}{\omega^{3}}\left(\frac{\mathrm{d} \omega}{\mathrm{d} t}\right)^{2}-\frac{2 \pi}{\omega^{2}} \frac{\mathrm{d}^{2} \omega}{\mathrm{d} t^{2}}
$$

and

$$
\frac{\mathrm{d} T}{\mathrm{~d} t}=-\frac{2 \pi}{\omega^{2}} \frac{\mathrm{d} \omega}{\mathrm{d} t}
$$

we may also write an adiabaticity condition relative to $\frac{\mathrm{d} T}{\mathrm{~d} t}$ assuming $\frac{\mathrm{d}^{2} \omega}{\mathrm{d} t^{2}}$ to be negligible as compared to $\frac{\mathrm{d} \omega}{\mathrm{d} t}$, then

$$
\left|T \frac{\mathrm{d}^{2} T}{\mathrm{~d} t^{2}}\right| \# 2\left(\frac{\mathrm{d} T}{\mathrm{~d} t}\right)^{2}
$$

the adiabaticity condition then writes

$$
\frac{2}{T^{2}}\left(\frac{\mathrm{d} T}{\mathrm{~d} t}\right)^{2}<4 \omega^{2}=4\left(\frac{2 \pi}{T}\right)^{2}
$$

or

$$
\frac{\mathrm{d} T}{\mathrm{~d} t}<2 \pi \sqrt{2}
$$

In ref. [7] we arrived qualitatively at an adiabaticity condition

$$
-\frac{\mathrm{d} T}{\mathrm{~d} t}<1
$$

It appears that this was much too restrictive. We recall that this condition was obtained by requiring that the variation of the period over one period be less than the period itself. We may account for part of the difference from the following considerations :

- The oscillatory motion of the variance $y=\sigma^{2}(Z)$ has a period $T / 2$ rather than $T$.

- It is only necessary for the system to achieve half an oscillation in order to span all values of the variance.

Taking these two remarks into account, equation (3.7) would have to be changed into

$$
\frac{\mathrm{d} T}{\mathrm{~d} t} \times \frac{T}{4}<T
$$

or

$$
\frac{\mathrm{d} T}{\mathrm{~d} t}<4
$$

which is not too far from condition (3.6).

4. Results and discussion. - Equation (3.4) has been solved assuming a linear decreases of $\omega^{2}(t)$ with time down to zero value at scission. Different rates of decrease have been tested. The system was initially assumed to be in its ground state and follow its modification adiabatically. Figure 1 shows the results obtained corresponding to a «necking " velocity

$$
\frac{\mathrm{d} c}{\mathrm{~d} t}=2 \text { Fermis } / 10^{-21} \mathrm{~s}
$$

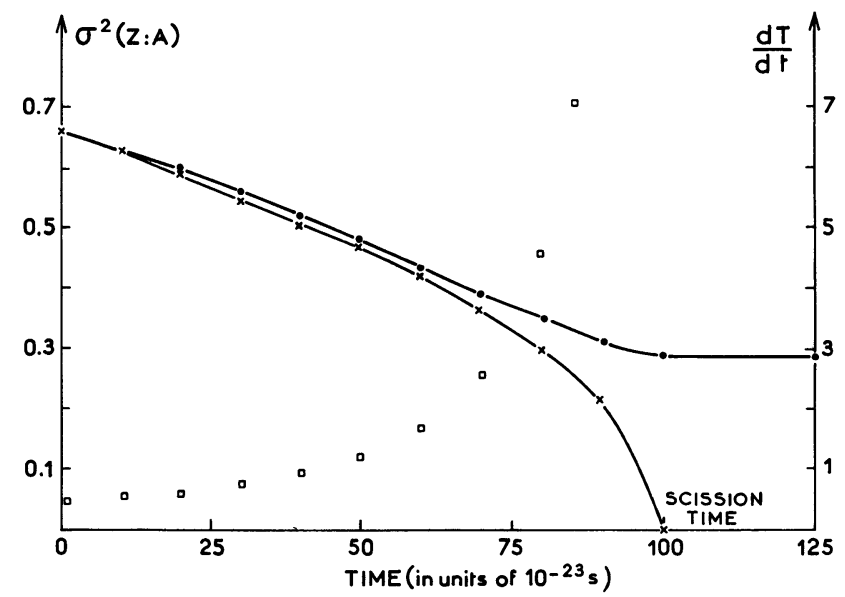

Fig. 1. - Isobaric Variance $\sigma^{2}(Z: A)$ as a function of time. - Results obtained after solving differential equation (3.4) with an initial neck radius of 2 Fermis and necking velocity of 2 Fermis $/ 10^{-21} \mathrm{~s}$. $\times$ Adiabatic solution : zero point width of the harmonic oscillator calculated for each value of the neck. $\square$ Adiabaticity parameter $\mathrm{d} T / \mathrm{d} t$.

The figure shows, as a function of time, the variance $\sigma^{2}(Z)$ as obtained from equation (3.4) as well as the adiabatic solution and the variation of the adiabaticity parameter $\mathrm{d} T / \mathrm{d} t$. 
The asymptotic value of $\sigma^{2}(Z)=0.29$ is very close from the experimental one. It is also seen that the value of the adiabaticity parameter reached when the adiabatic solution equals 0.29 is

$$
\frac{\mathrm{d} T}{\mathrm{~d} t}=5.5
$$

which is close from both estimates (3.6) and (3.8). One may therefore say that the isobaric distribution is frozen in when the adiabaticity parameter $\mathrm{d} T / \mathrm{d} t$ reaches a value around 6 .

Figure 2 shows the asymptotic value of $\sigma^{2}(Z)$ as a function of the necking velocity. The experimental value is also reported in figure. It can be seen that necking velocities between 1.5 and 4 Fermis $/ 10^{-21} \mathrm{~s}$ would account for the observed isobaric widths in this very simple model. Such values seem to be realistic. A much faster necking velocity would result almost certainly in a too large isobaric width and can therefore, safely, be ruled out. On the other hand a slower necking velocity would give a zero point width smaller than the experimental one. In that case one cannot, a priori, rule out a thermal excitation of the mode increasing the width above its zero point value. However the weak dependence of $\sigma^{2}(Z: A)$ over the fragments' excitation energies found

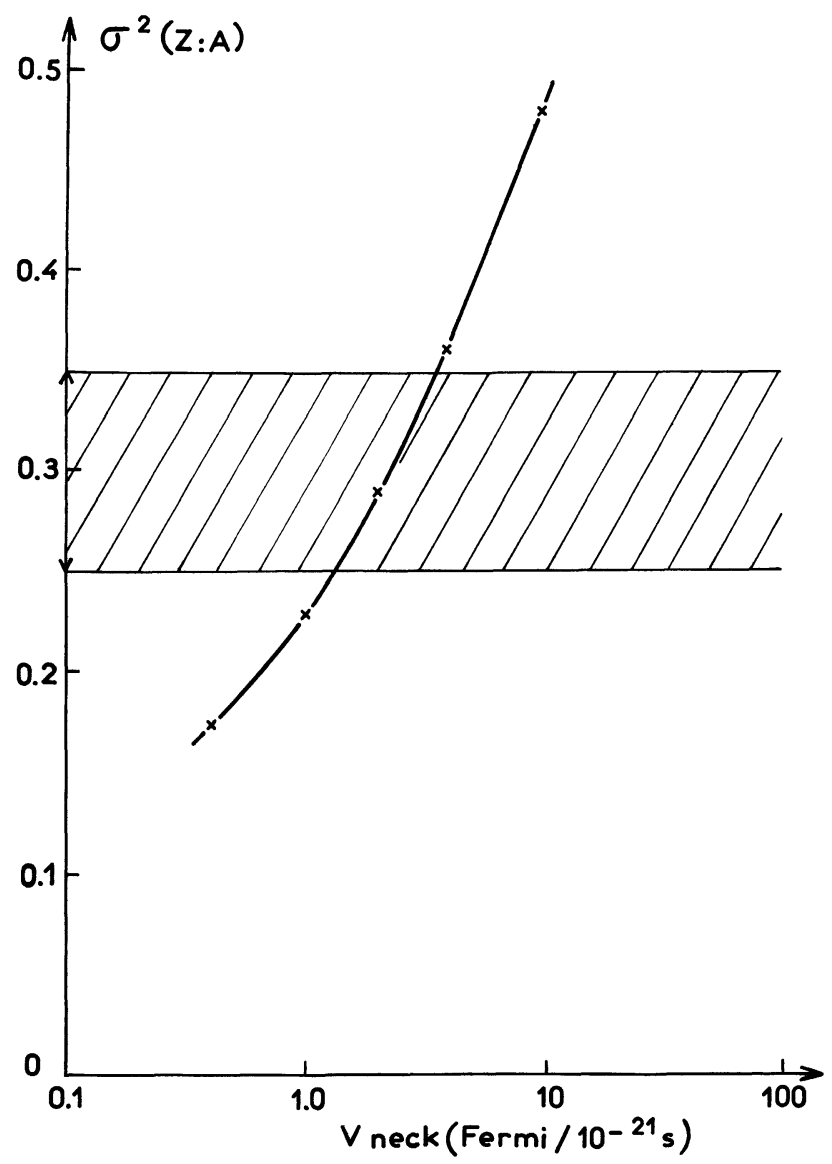

Fig. 2. - Calculated Isobaric Variance $\sigma^{2}(Z: A)$ as a function of necking velocity. The hatched zone corresponds to the possible experimental values including errors. by Clerc et al. [11] points very strongly against such a thermal excitation while it is easily explained in our simple frozen zero point distribution model.

For increasing excitation energies of the fissioning nucleus we would expect variation of $\sigma^{2}(Z: A)$ with the temperature $T_{\mathrm{s}}$ at saddle point

$$
\sigma^{2}(Z: A)=\frac{r}{K}\left(\frac{\hbar \omega_{\mathrm{s}}}{2}-\frac{\hbar \omega_{\mathrm{s}}}{\exp \frac{\hbar \omega_{\mathrm{s}}}{T_{\mathrm{s}}}-1}\right)
$$

$r$ is a reduction factor such that in the zero temperature case which we have examined above

$$
\sigma^{2}(Z: A)=r \frac{\hbar \omega_{\mathrm{s}}}{2 K}
$$

$\hbar \omega_{\mathrm{s}}$ and $T_{\mathrm{s}}$ are the eigen energy and temperature at saddle point.

This temperature dependence should only become noticeable for temperature of several $\mathrm{MeV}$. It may be hopeless to observe it unambiguously because of neutron evaporation by the highly excited fragments. Neutron evaporation may also prevent a test of the dynamical approach against the thermodynamical one, since in both cases the variations of $\sigma^{2}(Z: A)$ are relatively small.

Results such as those obtained by Clerc [11] and quoted above may remain the strongest experimental evidence in favor of quantal determination of isobaric distributions in fission.

As noted in (3.7) one should consider the mass degree of freedom on the same footing as the charge equilibration one. Due to the much lower eigenfrequency of the mass mode, however, it is probable that it is thermally excited. For the same reason the motion in the mass degree of freedom probably loses its adiabaticity earlier than the charge equilibration mode. One is therefore led to the concept of a dynamical " scission point " which would depend on the considered collective mode.

Finally we come back to the possible extension of the model to heavy ion reactions. This might prove difficult aside from the objection raised by L. Moretto [5]. In the initial state the two fragments are separated, and thus, the eigenfrequency of the charge equilibration mode vanishes. It is, therefore, not possible to assume adiabatic initial conditions. A zero width distribution would obey the differential equation (3.6) throughout the process. It is, however, not physical since it would require an infinite energy to keep it during the nuclear contact. This shows that it is probably necessary to treat more or less explicitly, the coupling to other degrees of freedom in the initial part of the reaction. For moderate or low energy heavy ion reaction it may be correct to assume some kind of statistical equilibrium close to the classical turning-point. In such a case an approach similar to the one just applied to fission may be 
justified, replacing the saddle point by the turning point. A close inspection of the condition for the validity of this approach might help understanding the present puzzling experimental situation where apparently contradictory results have been obtained for different systems [9], [12].
The author wants to aknowledge fruitful discussions with P. Schuck, R. Arvieu, C. Guet and L. Moretto. $\mathrm{He}$ is especially grateful to J. Randrup for oral communication of unpublished results concerning a somewhat different approach to the problems considered here.

\section{References}

[1] Gatty, B., Guerreau, D., Lefort, M., Pouthas, J., Tarrago, X., Galin, J., Cauvin, B., Girard, J. and Nifenecker, H., Z. Phys. A 273 (1975) 65.

[2] Galin, J., J. Physique Colloq. 37 (1976) C5-83.

[3] Grégoire, C., Lucas, R., N'Go, C. and Hofmann, H., IVth Balaton Conference on Nuclear Physics.

[4] Grégoire, C., Ballini, R., Cauvin, B., Fabbro, F., Girard, J., Lemaire, M. C., Mazur, C., Nifenecker, H., Peng, J. C., RibraG, M., International Workshop on gross properties of nuclei and nuclear excitations VII. Hirschegg, Austria (1979).

[5] Moretto, L., Lectures given at the International School of Nuclear Physics "Ettore Majorana " Center for Scientific Culture Erice, Sicily (1979).

[6] Nifenecker, H., Signarbieux, C., Babinet, R. and Poitou, J., Proceedings 3rd Symposium on Physics and Chemistry of Fission, Rochester (1973) Vol. II, p. 117.
[7] Nifenecker, H., Blachot, J., Bocouet, J. P., Brissot, R., Crançon, J., Hamelin, C., Mariolopoulos, G., Ristori, Ch., 4th IAEA Symposium on Physics and Chemistry of Fission, Jülich (1979).

[8] Brosa, U., Krappe, H. J., Z. Physik A 284 (1978) 65.

[9] Berlanger, M., GobBi, A., Hanappe, F., Lynen, U., N'Go, C., Olmi, A., SanN, H., Stelzer, N., Richel, H., and Rivet, M. F. Z. Phys. A 291 (1979) 133.

[10] Messiah, A., Mécanique quantique I (1959) 184.

[11] Clerc, H. G., Lang, W., Wohlfarth, H., Schrader, H. and SCHMIDT, K. H., 4th IA EA International Symposium on Physics and Chemistry of Fission, Jülich (1979).

[12] Wirth, G., Brüchle, W., Gäggeler, H., Kratz, J. V., Schädel, M., Warnecker, I., Hermann, G., Weis, M., LUCAS, R., PoITOU, J., same as ref. [4]. 